\title{
A self-consistent, relativistic implementation of the LSDA+DMFT method
}

\author{
J. Minár ${ }^{1, a}$, H. Ebert ${ }^{2}$, and L. Chioncel ${ }^{3,4}$ \\ 1 New Technologies-Research Center, University of West Bohemia, Univerzitni 8, 30614 \\ Pilsen, Czech Republic \\ 2 Department Chemie, Physikalische Chemie, Universität München, Butenandtstrasse \\ 5-13, 81377 München, Germany \\ 3 Theoretical Physics III, Center for Electronic Correlations and Magnetism, Institute of \\ Physics, University of Augsburg, 86135 Augsburg, Germany \\ 4 Augsburg Center for Innovative Technologies, University of Augsburg, 86135 Augsburg, \\ Germany
}

Received 10 February 2017 / Received in final form 27 March 2017

Published online 10 July 2017

\begin{abstract}
In this review we report on developments and various applications of the combined Density Functional and Dynamical Mean-Field Theory, the so-called LSDA + DMFT method, as implemented within the fully relativistic KKR (Korringa-Kohn-Rostoker) band structure method. The KKR uses a description of the electronic structure in terms of the single-particle Green function, which allows to study correlation effects in ordered and disordered systems independently of its dimensionality (bulk, surfaces and nano-structures). We present self-consistent LSDA+DMFT results for the ground state and spectroscopic properties of transition metal elements and their compounds. In particular we discuss the spin-orbit induced orbital magnetic moments for $\mathrm{Fe}_{x} \mathrm{Ni}_{1-x}$ disordered alloys, the magnetic Compton profiles of fcc $\mathrm{Ni}$ and the angle-resolved photoemission spectroscopy (ARPES) spectra for gallium manganese arsenide dilute magnetic semiconductors. For the (GaMn)As system a direct comparison with the experimental ARPES spectra demonstrates the importance of matrix element effects, the presence of the semi-infinite surface and the inclusion of layer-dependent self-energies.
\end{abstract}

\section{Introduction}

During the last decade the Density Functional Theory, through its various exchange correlations functionals such as the local spin density approximation (LSDA), has been succesfully combined with the Dynamical Mean-Field Theory (DMFT). This combination came to be known as the LSDA + DMFT scheme and turned out to be very successful in dealing with the electronic structure of a wide range of correlated materials. Meanwhile, various LSDA + DMFT implementations are available [1-3].

\footnotetext{
${ }^{a}$ e-mail: jminar@ntc.zcu.cz
} 
In the early LSDA+DMFT implementations a two step procedure has been used. In the first step the LSDA problem was solved using an effective one-particle Kohn-Sham Hamiltonian and the single particle wave-functions (Kohn-Sham basis set) integrated into the Density Functional variational approach. The corresponding Green function is obtained using the spectral representation of the Kohn-Sham Hamiltonian. In the second step, the interacting problem, the low-energy effective Hamiltonian was formulated within a Wannier-like basis obtained through downfolding or, alternatively, by a suitable combination of Kohn-Sham basis sets. This low-energy Hamiltonian was solved using DMFT, resulting in local self-energies and Green functions that can in turn be used to calculate new charge densities and new LSDA potentials. The above two step procedure follows partly the spirit of the spectral density functional theory (SDFT) proposed by Savrasov and Kotliar [4]. The key idea of SDFT, however, is the self-consistent procedure applied to the Dyson equation. This leads to the quasiparticle Schrödinger (or Dirac) equation with a non-hermitian (component in the) Hamiltonian. An elegant way around the technical difficulties of the SDFT and the Hamiltonian formulation is provided by the multiple scattering KKR method.

In this manuscript we briefly review the fully self-consistent (with respect to charge density and self energy) LSDA+DMFT implementation within the full potential fully relativistic multiple-scattering KKR method [3,5]. A more extended account for the recent developments using the KKR method has already been published [6]. It is important to note the KKR flexibility in using the Dyson equation to relate the Green function of a perturbed system to the Green function of a suitable unperturbed reference system. It allows in particular to calculate the properties of low dimensional systems, e.g., semi-infinite 2D surfaces, nanostructures and embedded $3 \mathrm{D}$ or $2 \mathrm{D}$ systems, without using an artificial supercell construction. Additionally, the KKR Green function method allows to treat disorder within the Coherent Potential Approximation (CPA) for substitutional disordered alloys [5,7]. Finally, in combination with DMFT, all the above problems can be addressed together with the electronic correlations. What makes relativistic multiple-scattering KKR particularly unique is the use of a fully relativistic formulation on the basis of the fourcomponent Dirac formalism. Important consequences derive from the presence of all aspects of relativistic effects, contrary to most frequent implementations which consider only spin-orbit coupling. Therefore in combination with the LSDA+DMFT, the simultaneous description of the orbital magnetic moments [8-10], relativistic band structure [11], hyperfine fields [12], spin-fluctuations [13], and accurate total energy calculations [14] becomes possible. In the later years, this method was successfully applied to a variety of disordered magnetic systems including transition metal $[9,15]$, Heusler alloys [16,17] and diluted magnetic semi-conductors systems [18,19]. Furthermore, correlation effects on the hcp- $\mathrm{Co}(0001)$ surface have been studied and showed pronounced changes in the band structure seen when the magnetisation is oriented in or out-of-plane, as a consequence of the spin-orbit coupling acting on the electronic states [20]. Similar important correlation effects in the band structure close to the Fermi level have been found for Fe, Co and Ni surfaces [21]. The analysis of ground state properties have been complemented with spectroscopy investigations, in particular using the angle-resolved photoemission (ARPES). The commonly used comparison between the calculated density of states / spectral functions and the corresponding measured spectra ignores among several things the matrix elements and surface effects. Within the KKR method all these effects are included to a very large extent. For example, an advanced analysis of ARPES data combines coherently the LSDA + DMFT method with the one step model of photoemission and allows to account properly for all relevant issues: the surface geometry, time-reversed LEED state (as the best possible representation of the final state) and most importantly the 
transition matrix elements. These recent theoretical developments have been summarised in two recent reviews $[3,22]$ and were made possible within this research unit.

In the following we shall review several results obtained with the LSDA + DMFT method as implemented within the fully relativistic KKR method. In Section 2 we briefly review the formalism. The results of calculations for the ground state properties of disordered $\mathrm{Fe}_{x} \mathrm{Ni}_{1-x}$ alloys are presented in Section 3, while spectroscopy related results are shown in Section 4. In particular in Section 4.1 we discuss the results of the Compton profiles of $\mathrm{Ni}$ and Section 4.2 presents the results for the angleintegrated as well as angle resolved photoemission spectra of transition metal surfaces and disordered materials calculated within the one-step model of photoemission.

\section{Computational details}

When calculating the electronic structure of a solid, electron correlations may be accounted for in a rather general way by complementing the scalar, real and local single-particle potential $V_{\mathrm{LSDA}}(\mathbf{r})$ as provided for example by LSDA by a suitably chosen self energy $\Sigma\left(\mathbf{r}, \mathbf{r}^{\prime}, E\right)$ that in the most general case is energy dependent, complex and non-local. A very powerful way to solve the electronic structure problem for this situation is to solve the corresponding Dyson equation for the single-particle Green function $G\left(\mathbf{r}, \mathbf{r}^{\prime}, E\right)$ :

$$
\begin{aligned}
G\left(\mathbf{r}, \mathbf{r}^{\prime}, E\right)= & G_{0}\left(\mathbf{r}, \mathbf{r}^{\prime}, E\right)+\int d^{3} r^{\prime \prime} \int d^{3} r^{\prime \prime \prime} G_{0}\left(\mathbf{r}, \mathbf{r}^{\prime \prime}, E\right)\left[V_{\mathrm{LSDA}}\left(\mathbf{r}^{\prime \prime}\right) \delta\left(\mathbf{r}^{\prime \prime}-\mathbf{r}^{\prime \prime \prime}\right)\right. \\
& \left.+\Sigma\left(\mathbf{r}^{\prime \prime}, \mathbf{r}^{\prime \prime \prime}, E\right)\right] G\left(\mathbf{r}^{\prime \prime \prime}, \mathbf{r}^{\prime}, E\right)
\end{aligned}
$$

where $G_{0}\left(\mathbf{r}, \mathbf{r}^{\prime}, E\right)$ is the electronic Green function for the reference system and $\mathbf{r}, \mathbf{r}^{\prime}$ are defined relative to the center of an atomic cell corresponding to a specific site. The reference system is described by a one-electron Hamiltonian with non-overlaping potentials located on the regular lattice. The non-overlaping potentials are necessary for the rigurous applications of the multiple scattering. The Green function for electrons propagating in the potential of the type assumed in equation (1) is obtained solving the single-site and subsequently the multiple scattering problem. These steps are briefly described below.

\subsection{The single-site problem}

Adopting a fully relativistic formulation the single-site problem is specified by the corresponding single-site Dirac equation:

$$
\left[\frac{\hbar}{i} c \boldsymbol{\alpha} \cdot \nabla+\beta m c^{2}+\bar{V}(\mathbf{r})+\beta \sigma_{\mathrm{z}} \cdot B_{\mathrm{eff}}(\mathbf{r})-E\right] \Psi(\mathbf{r})+\int d^{3} r^{\prime} \Sigma\left(\mathbf{r}, \mathbf{r}^{\prime}, E\right) \Psi\left(\mathbf{r}^{\prime}\right)=0,
$$

where all quantities have their usual meaning [23] and $\bar{V}(\mathbf{r})$ and $\beta \sigma_{\mathrm{z}} \cdot B_{\text {eff }}(\mathbf{r})$ are the spin independent and spin dependent parts of the LSDA potential, respectively, restricted - as the self energy $\Sigma\left(\mathbf{r}, \mathbf{r}^{\prime}, E\right)$ - to the atomic cell centered at site $n$.

Equation (2) may be solved by making an ansatz for the wave function $\Psi(\mathbf{r})$ in terms of four-component partial waves of the form [24]

$$
\psi_{\nu}(\mathbf{r}, E)=\sum_{\Lambda} \psi_{\Lambda \nu}(\mathbf{r}, E)
$$


with

$$
\psi_{\Lambda \nu}(\mathbf{r}, E)=\left(\begin{array}{c}
g_{\Lambda \nu}(r, E) \chi_{\Lambda}(\hat{\mathbf{r}}) \\
i f_{\Lambda \nu}(r, E) \chi_{-\Lambda}(\hat{\mathbf{r}})
\end{array}\right),
$$

where the index $\Lambda=(\kappa, \mu)$ represents the relativistic spin-orbit and magnetic quantum numbers $\kappa$ and $\mu$, respectively, while the index $\nu$ labels the independent solutions. In equation (4) $g_{\Lambda \nu}(r, E)$ and $f_{\Lambda \nu}(r, E)$ represent the large and small components, respectively, and $\chi_{\Lambda}(\hat{\mathbf{r}})$ is a bi-spinor [23]. This way one ends up with a coupled set of integro-differential equations for the radial functions $g_{\Lambda \nu}(r, E)$ and $f_{\Lambda \nu}(r, E)$. After these equations have been solved by a suitable scheme [5] the corresponding singlesite $t$-matrix $t_{\Lambda \Lambda^{\prime}}(E)$ is obtained by matching the wave functions smoothly to the free electron solution at the boundary of the atomic cell [25].

\subsection{Multiple-scattering path-operator and the Green function}

Having accounted for the local self energy $\Sigma\left(\mathbf{r}, \mathbf{r}^{\prime}, E\right)$ already when solving the single site problem all remaining steps to get the Green function are just the same as for plain LSDA-based calculations. Within the multiple scattering formalism used in the KKR method the Dyson equation (1) for the Green function is solved using the equation of motion for the so-called scattering-path operator $\underline{\tau}^{n n^{\prime}}(E)$ connecting sites $n$ and $n^{\prime}[26]$ :

$$
\underline{\tau}^{n n^{\prime}}(E)=\underline{t}^{n}(E) \delta_{n n^{\prime}}+\underline{t}^{n}(E) \sum_{m \neq n} \underline{G}_{0}^{n m}(E) \underline{\tau}^{m n^{\prime}}(E),
$$

where the underscore indicates matrices with respect to the spin-angular $\Lambda$ representation and $\underline{G}_{0}^{n m}(E)$ stands for the free electron Green function or the real space KKR structure constant matrix, respectively. Dealing with a three-dimensional periodic system equation (5) can be solved exactly by Fourier transformation leading to $[27]$ :

$$
\underline{\tau}^{n n^{\prime}}(E)=\frac{1}{\Omega_{\mathrm{BZ}}} \int_{\Omega_{\mathrm{BZ}}} d^{3} k\left[\underline{t}(E)^{-1}-\underline{G}_{0}(\mathbf{k}, E)\right]^{-1} e^{i \mathbf{k}\left(\mathbf{R}_{n}-\mathbf{R}_{n^{\prime}}\right)},
$$

where $G_{0}(\mathbf{k}, E)$ is the standard $\mathbf{k}$-dependent KKR structure constant matrix.

With the multiple scattering problem solved the Green function $G\left(\mathbf{r}, \mathbf{r}^{\prime}, E\right)$ can finally be written as $[28,29]$ :

$$
\begin{aligned}
G\left(\mathbf{r}, \mathbf{r}^{\prime}, E\right)= & \sum_{\Lambda \Lambda^{\prime}} Z_{\Lambda}^{n}(\mathbf{r}, E) \tau_{\Lambda \Lambda^{\prime}}^{n n^{\prime}}(E) Z_{\Lambda^{\prime}}^{n^{\prime} \times}\left(\mathbf{r}^{\prime}, E\right) \\
& -\sum_{\Lambda}\left[Z_{\Lambda}^{n}(\mathbf{r}, E) J_{\Lambda}^{n \times}\left(\mathbf{r}^{\prime}, E\right) \Theta\left(r^{\prime}-r\right)\right. \\
& \left.+J_{\Lambda}^{n}(\mathbf{r}, E) Z_{\Lambda}^{n \times}\left(\mathbf{r}^{\prime}, E\right) \Theta\left(r-r^{\prime}\right)\right] \delta_{n n^{\prime}},
\end{aligned}
$$

where the regular and irregular wave functions, $Z_{\Lambda}^{n}(\mathbf{r}, E)$ and $J_{\Lambda}^{n}(\mathbf{r}, E)$, respectively, are normalised in an appropriate way $[28,29]$. Here it should be stressed that for a complex non-Hermitian self energy $\Sigma\left(\mathbf{r}, \mathbf{r}^{\prime}, E\right)$ one has to distinguish between the leftand right-hand side solutions to the single-site Dirac equation (2) [30]. Accordingly, the left-hand side solutions $Z_{\Lambda}^{n \times}(\mathbf{r}, E)$ and $J_{\Lambda}^{n \times}(\mathbf{r}, E)$ have been indicated by the symbol $\times$. 
From the knowledge of the Green function, properties of the system represented by an operator $\mathcal{A}$ are obtained by evaluating the expression

$$
\langle\mathcal{A}\rangle=-\frac{1}{\pi} \Im \int^{E_{\mathrm{F}}} d E \int d^{3} r \operatorname{Trace} \mathcal{A} G(\mathbf{r}, \mathbf{r}, E),
$$

where the energy integration extends over the range of the occupied part of the valence band up to the Fermi energy $E_{\mathrm{F}}$. For instance the spin and orbital magnetic moments are computed with equation (8) using for $\mathcal{A}$ the operators $\mu_{\mathrm{B}} \beta \sigma_{\mathrm{z}}$ and $\mu_{\mathrm{B}} \beta l_{\mathrm{z}}$, respectively.

\section{Ground state properties}

One of the most appealing features of the KKR method is the possibility to link directly to the CPA alloy theory. Dealing with a binary alloy of A and B atoms the corresponding CPA medium is determined by the requirement that the substitutional embedding of an A or B atom into the CPA medium does not lead in the average to additional scattering. This demand is most easily expressed in terms of the scattering path operator for $\alpha=\mathrm{A}, \mathrm{B}$ :

$$
x_{\mathrm{A}} \underline{\tau}_{\mathrm{A}}^{n n}+x_{\mathrm{B}} \underline{\tau}_{\mathrm{B}}^{n n}=\underline{\tau}_{\mathrm{CPA}}^{n n}
$$

with the component projected scattering path operator

$$
\underline{\tau}_{\alpha}^{n n}=\underline{\tau}_{\mathrm{CPA}}^{n n}\left[1+\left(\underline{t}_{\alpha}^{-1}-\underline{t}_{\mathrm{CPA}}^{-1}\right) \underline{\tau}_{\mathrm{CPA}}^{n n}\right]^{-1} .
$$

Obviously, equations (6), (9) and (10) have to be solved for the single-site $t$-matrix and scattering path operator, $\underline{t}_{\mathrm{CPA}}$ and $\underline{\tau}_{\mathrm{CPA}}^{n n}$, respectively, of the CPA medium. As emphasised by Drchal et al. [7] assuming the self energy $\Sigma\left(\mathbf{r}, \mathbf{r}^{\prime}, E\right)$ to be site-diagonal as it is done within the LSDA $+\mathrm{U}$ as well as within standard DMFT schemes allows to determine the effective CPA mean-field medium, i.e., to perform the single-site CPA configurational average as for plain LSDA-based calculations.

In this section we present as an example the CPA formalism applied to the $\mathrm{Fe}_{x} \mathrm{Ni}_{1-x}$ alloy system. These alloys show very interesting magnetic properties. Permalloy $(x=0.19)$ which has fcc structure, shows vanishing magnetic anisotropy. On the other hand, the bcc invar alloy $(x=0.65)$ have been intensively studied for low magnetic moment and low thermal expansion. Using this example we will show that the self energy shows non trivial behaviour w.r.t. concentration and we will discuss the very well known problem of underestimation of the orbital magnetic moments by spin density functional theory (for further discussions concerning electronic structure and hyperfine fields see [12]).

Using the relativistic KKR-CPA formalism outlined above the electronic structure of ferromagnetic fcc-Fe $\mathrm{Fe}_{x} \mathrm{Ni}_{1-x}$ alloys has been determined in a self-consistent way using experimental lattice parameters. LSDA-based calculations have been done using the parametrisation of Vosko et al. [31] for the exchange-correlation potential. For the complementary self-consistent LSDA+DMFT calculations the parameters $U=1.8 \mathrm{eV}, J=0.9 \mathrm{eV}$ for $\mathrm{Fe}$ and $U=2.8 \mathrm{eV}, J=0.9 \mathrm{eV}$ for $\mathrm{Ni}$, respectively, have been used. These parameters have been found to give the best agreement between various experimental properties (e.g., spectroscopy and orbital magnetic moments) and LSDA + DMFT calculations for the pure elements [3]. For the DMFT-part of the LSDA + DMFT calculations a FLEX-solver has been used [32] working on a Matsubara energy grid corresponding to a temperature of $400 \mathrm{~K}$. Double counting (DC) was corrected using the around mean-field (AMF) 

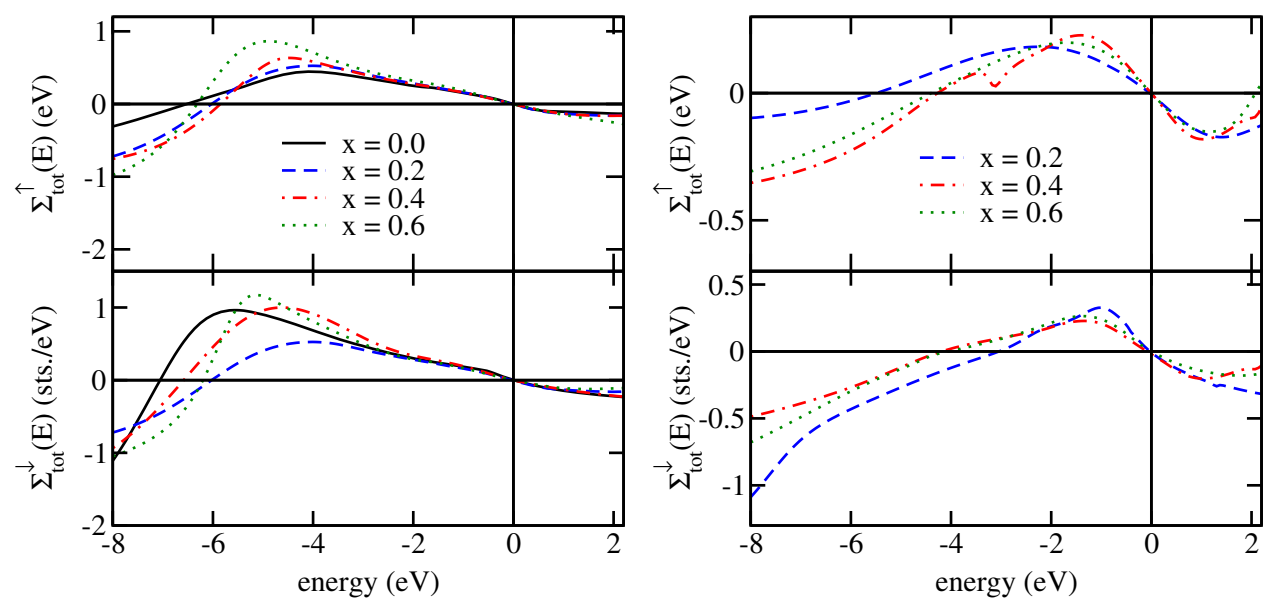

Fig. 1. Spin resolved and orbital averaged DMFT real part of the self energy for Ni (left panel) and $\mathrm{Fe}$ (right panel) for fcc- $\mathrm{Fe}_{x} \mathrm{Ni}_{1-x}$ disordered alloy as calculated within the KKRCPA formalism. Figure reproduced from [12]. (This figure is subject to copyright protection and is not covered by a Creative Commons license.)
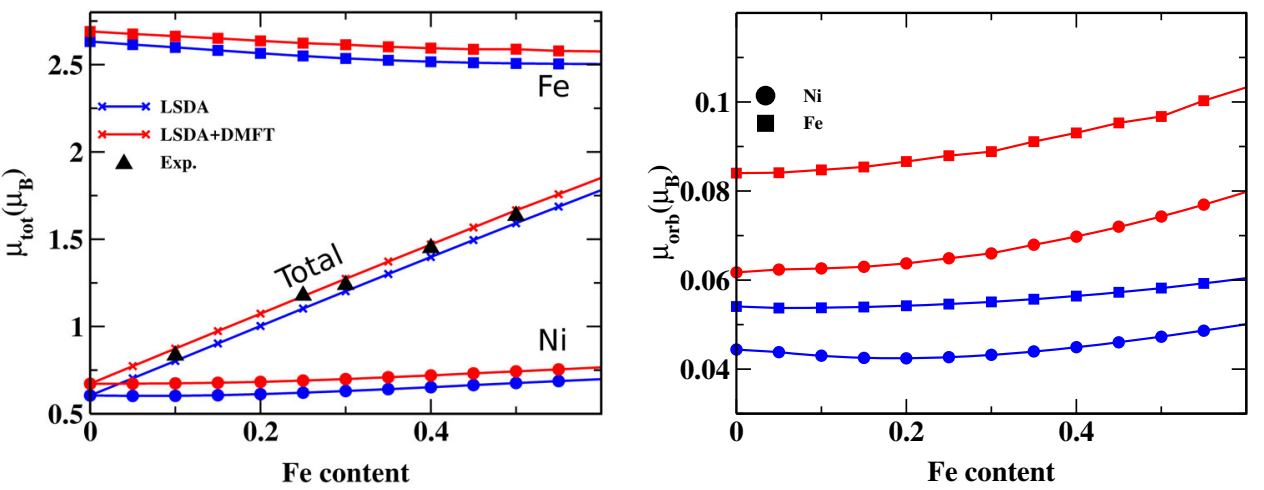

Fig. 2. Left panel: element resolved and total magnetic moments of the fcc- $\mathrm{Fe}_{x} \mathrm{Ni}_{1-x}$ disordered alloy calculated within the LSDA (blue) and LSDA + DMFT (red) approach. Experimental data taken from [33]. Right panel: element resolved contributions to the orbital magnetic moment (LSDA (blue) and LSDA + DMFT (red)). In both panels circles represent $\mathrm{Ni}$ and squares Fe contributions, respectively.

scheme (for details concerning DC corrections within KKR calculations see reference $[3,8]$ ). In Figure 1 , the real part of the spin-dependent self energy as a function of the concentration is shown. It should be mentioned that only the orbital averaged dynamical part of the self energy is shown. The order of magnitude of the self energy reflects the very different $U$-values appropriate for Fe and Ni. For both components, the self energy varies with concentration in an appreciable and not trivial way. The imaginary part of the self energy (not shown here) shows a similar behaviour. In addition one notes that the broadening of the spectral features (not shown here) due to the imaginary part of self energy is comparable to the disorder induced broadening.

As it was mentioned above, the magnetic properties of the $\mathrm{Fe}_{x} \mathrm{Ni}_{1-x}$ alloy are of great interest. Only recently Glaubitz et al. [33] could obtain element resolved spin and orbital moments for the whole concentration region by means of X-ray magnetic circular dichroism. In the left panel of Figure 2 we compare element resolved total 
magnetic moments as calculated by LSDA and LSDA + DMFT method. In both cases, the total magnetic moment follow the Slater-Pauling curve [34], with a magnetic moment that drops continuously from $\mathrm{Fe}$ to Ni. This seems to reflect the continuous filling of the $3 d$ band. However, as clearly confirmed by the XCMD results, Fe and $\mathrm{Ni}$ keep their individual properties in contrast to this naive rigid band-like picture. The LSDA + DMFT magnetic moments are slightly bigger than LSDA results. As shown in the right panel of Figure 2, this enhancement can be traced back to the higher orbital magnetic moments obtained by the LSDA + DMFT. Orbital magnetic moments are caused by spin-orbit coupling and are typically underestimated within the framework of plain spin density functional theory. To improve the description of orbital magnetism, several related approaches have been proposed. The most general approach which would be based on the current density functional theory is until now, due to missing current exchange correlation functionals, impracticable. It has been realised, that the explicit account of local many-body correlations by means of LSDA $+\mathrm{U}$ can alternatively solve this problem $[35,36]$. The explicit account for local correlations within the LSDA + DMFT approach which includes Hartree-Fock like contributions to the self energy, should improve simultaneously the description of the orbital magnetic moments [8,9], the relativistic band structure [11] as well as spectral properties $[10,15,20,21,37-43]$. Concerning this, a very important issue is an appropriate treatment of the so-called double counting terms. Comparison of the calculated results directly with experimental data for the orbital magnetic moment for $\mathrm{Fe}_{x} \mathrm{Ni}_{1-x}$ clearly shows that using the AMF setting is most adequate for this class of materials.

\section{LSDA+DMFT for calculations of spectroscopic properties}

Spectroscopy is an extremely important experimental tool providing information on the electronic structure of the probed system that has to be seen as a stringent benchmark for the success of any electronic structure theory. Photoemission spectroscopy (PES) or its inverse - the Bremsstrahlen isochromat spectroscopy (BIS) - in their angle-integrated form should reflect the density of states (DOS) rather directly - in particular in the high photon energy regime (XPS). For that reason it is quite common to check the DMFT-based calculations by comparing the calculated DOS directly to PES spectra (see the reviews $[1,2,44]$ for examples). However, this approach ignores the influence of the specific PES matrix elements that in general will introduce an element- and energy-dependent weight to the partial DOS. In case of angle-resolved photoemission (ARPES) the situation is even move severe as the surface as well as dipole selection rules may have a very pronounced impact on the spectra [45] demanding for a coherent description as provided by the one-step model of photoemission $[22,46]$.

While ARPES has been succesfully used to map the spectral function (dispersion of bands and k-resolved density of states) as explained above, relatively little attention has been given to the wave function related quantities, despite the fact that wave function information may provide a more sensitive way of testing the theoretical models. The wave function can hardly be measured directly, instead the spectral momentum density which within the independent particle model is proportional to the square of the one-electron wave function can be obtained from scattering experiments. According to its basic definition the Compton scattering is an incoherent, inelastic scattering of a photon with a single electron. In the scattering process the photon transfers energy to the electron and the degree of energy loss is dependent on the scattering angle. The Compton scattering may provide complementary information 
to ARPES, in particular about the electronic correlations as we shall present below results from our recent publications $[43,47,48]$.

\subsection{Compton profiles}

The single particle momentum density of an interacting electronic system can be measured rather directly by high energy Compton scattering experiments [49]. Although the momentum density is a relatively simple function it incorporates in a non-trivial way the many-body aspects of the interactions between the electrons of the system. Of particular interest in the case of metals are the tails of the momentum density of conduction electrons which one would expect to extent beyond the Fermi surface because of electron-electron correlations and the higher momentum components of the conduction electron Bloch functions produced by the crystal potential. According to its definition, the Compton profile is a directional quantity, which represents the projection of the momentum density along the transfered momentum $\mathbf{K}=\mathbf{k}_{\text {in }}-\mathbf{k}_{\text {out }}$, with $\mathbf{k}_{\text {in (out) }}$ the k-vector for in(out)-going beam, which typically can be chosen to be parallel along certain crystallographic direction [hkl].

The KKR Green function formalism allows to compute Compton profiles $J_{\mathbf{K}}\left(p_{z}\right)$ and magnetic Compton profiles $J_{m a g, \mathbf{K}}\left(p_{z}\right)$ (MCPs) in a straightforward way $[50,73,74]$. In the case of a magnetic sample, the spin resolved momentum densities are computed within the framework of LSDA(+U/DMFT) approaches using the Green functions in momentum space, as follows:

$$
n_{m_{s}}(\mathbf{p})=-\frac{1}{\pi} \Im \int_{-\infty}^{E_{F}} G_{m_{s}}^{L S D A(+U / D M F T)}(\mathbf{p}, \mathbf{p}, E) d E
$$

where $m_{s}=\uparrow(\downarrow)$. The total electron $\left(n_{\uparrow}(\mathbf{p})+n_{\downarrow}(\mathbf{p})\right)$ and spin $\left(n_{\uparrow}(\mathbf{p})-n_{\downarrow}(\mathbf{p})\right)$ momentum densities projected onto the direction $\mathbf{K}$ defined by the scattering vector, allows to define the (magnetic) Compton profile as a double integral in the momentum plane perpendicular to the scattering momentum $\mathbf{p}_{z} \| \mathbf{K}$ :

$$
\begin{aligned}
J_{\mathbf{K}}^{L S D A(+U / D M F T)}\left(p_{z}\right) & =\iint\left[n_{\uparrow}(\mathbf{p})+n_{\downarrow}(\mathbf{p})\right] d p_{x} d p_{y} \\
J_{m a g, \mathbf{K}}^{L S D A(+U / D M F T)}\left(p_{z}\right) & =\iint\left[n_{\uparrow}(\mathbf{p})-n_{\downarrow}(\mathbf{p})\right] d p_{x} d p_{y} .
\end{aligned}
$$

\subsubsection{Compton difference profile}

The anisotropy or difference profile, i.e., the difference between two Compton profiles taken along different directions (for example $\mathbf{K} \|$ [110] and $\mathbf{K}^{\prime} \|[100]$ )

$$
\Delta J_{\mathbf{K}, \mathbf{K}^{\prime}}(p)=J_{110}(p)-J_{100}(p)
$$

is a frequently studied quantity that allows to eliminate to a large extent the isotropic contribution of the core electrons. Although the computed anisotropy has in general a trend similar to the experimental spectra, it often displays larger amplitudes of oscillations in comparison with the measured profiles. For several transition metal elements such discrepancies are found in the low momentum region $(\mathrm{Fe}, \mathrm{Ni}, \mathrm{V}, \mathrm{Cr})$ $[43,47,51-55]$. The amplitudes of the characteristic oscillations are determined by details of the fine structures of the momentum densities. In order to address these discrepancies, Lam and Platzmann $[56,57]$ introduced a correction related to the 
difference between the occupation function for a non-interacting $n^{\text {free }}(\mathbf{p})$ and a homogeneous interacting $n^{\text {int }}(\mathbf{p})$ electron gas. The Lam-Platzman correction acts in the low-momentum region and for some cases it reduces the differences between experiment and theory. Nevertheless, the theoretical values still overestimate the amplitude with respect to the experiment in the low momentum region and in addition the residual differences appear anisotropic, contradicting the isotropic correction of Lam and Platzmann. The anisotropic effects were modeled for $\mathrm{V}$ and $\mathrm{Cr}$ also by introducing an energy dependent occupation function for the $d$-orbitals [58]. While such corrections brought the theoretical profile in better agreement with the experiment, one has to stress that this has been achieved by incorporating the corrections empirically into the calculations.

Later on Bauer [59,60] suggested that inclusion of electron-electron correlation effects may improve the difference between the theoretical and the experimental profiles. Kubo [61] computed the occupation number density within the GW approximation and discussed the corrections to the Compton profile for the principal directions, concluding that the strong directional differences are due to the $d$-bands. Alternatively DMFT can be used to account for the electronic correlations. In addition to the anisotropy profiles according to equation (12) we studied $[43,47]$ the difference profiles taken along the same momentum direction with and without including electronic correlations:

$$
\Delta J_{\mathbf{K}}^{+U / D M F T}(p)=J_{\mathbf{K}}^{L S D A+(U / D M F T)}(p)-J_{\mathbf{K}}^{L S D A}(p) .
$$

In particular this quantity allowed us to discuss the momentum space anisotropy of correlations effects.

Comparison between theoretical and experimental amplitudes of the Compton profile anisotropies for $\mathrm{Ni}$ are presented and discussed in detail in references [62-64]. Our results [43,47] for the computed anisotropy profiles $J_{[110]}-J_{[001]}$ and $J_{[111]}-J_{[001]}$ are shown in Figure 3 . In the upper panels (both left and right columns) the LSDA results are presented, while in the middle and lower panel the spectra obtained using LSDA $+\mathrm{U}$ and DMFT can be seen. A very similar behaviour, independent of the various level of sophistication to include the Coulomb interaction is visible. In the lower panel (c) we compare the anisotropy spectra with the corresponding experimental data of Anastassopoulos et al. [64]. There is a rather satisfying agreement between theory and experiment, in particular for the difference $J_{[110]}-J_{[001]}$ (Fig. 3 , red lines) where the theoretical calculation properly reproduce most of the maxima and minima seen in the experiment. On the other hand for $J_{[111]}-J_{[001]}$ (Fig. 3 , green lines) differences may be seen not only in the amplitude of the oscillation but also in the position of the minima/maxima. The right column of Figure 3 shows the comparison on a reduced momentum scale $0<p_{z}<1 \mathrm{a}$.u. The LSDA results are seen to overshoot in the range $0<p_{z}<0.2$ a.u. the experimental spectra plotted with dashed lines.

In the same momentum range the LSDA+U results underestimate the experimental data as seen in Figure 3b. Figure 3c shows the LSDA + DMFT results and one can see that the dynamic correlations capture at best the behaviour of anisotropy of the Compton profile in the region around the zero momentum $p_{z}<0.2$ a.u. Previous analysis $[64,65]$ discussed qualitatively the discrepancies with the experimental spectral based on the shortcomings of the DFT exchange-correlation potentials in misplacing the position of the $d$-bands (orbitals). It is well known that LSDA overestimate the exchange splitting, and the static corrections using LSDA + U enhance the exchange splitting further. Therefore the $(+\mathrm{U})$ addition does not correct upon the position of the $d$-bands. On the contrary, LSDA + DMFT improves on the exchange splitting as a consequence of a Fermi-liquid type of self energy and we equally see in the panel (c) of Figure 3, although in a narrow momentum region $0<p_{z}<0.2$ a.u., 

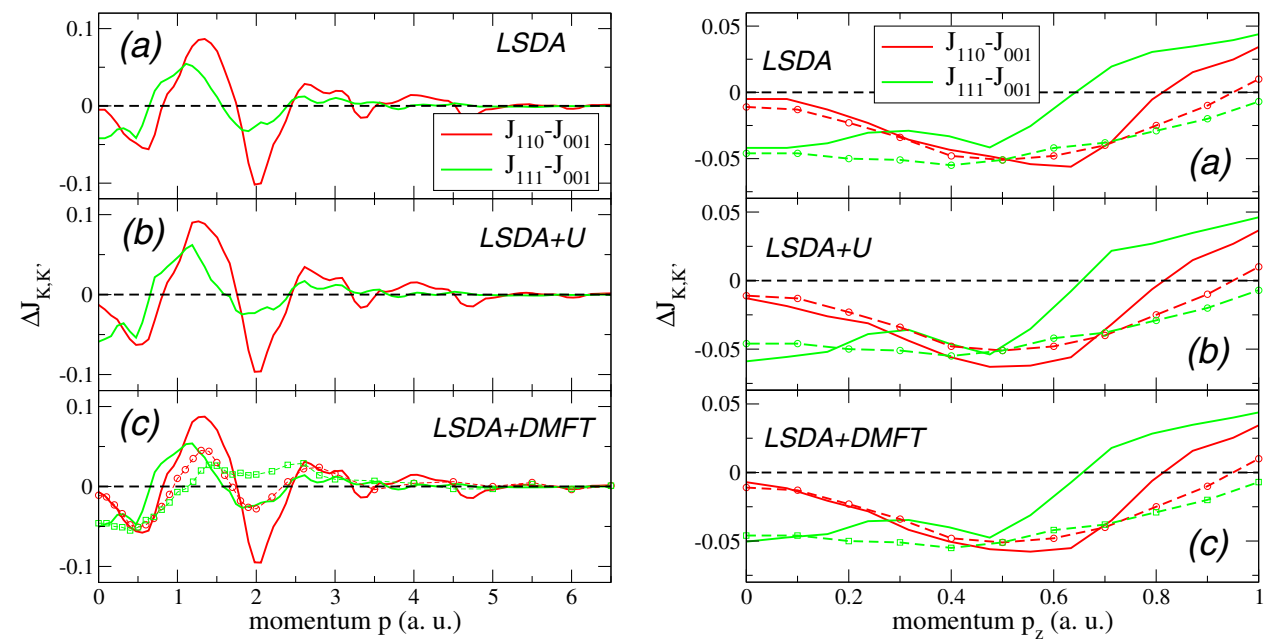

Fig. 3. Theoretical Compton profile anisotropies of Ni. Panel (a) gives LSDA anisotropies while panels (b) and (c) show the LSDA+U and LSDA + DMFT results for $U=2.3 \mathrm{eV}$, $J=0.9 \mathrm{eV}$ and $T=400 \mathrm{~K}$. Comparison with the experimental anisotropy is presented in panel (c). Right column: enlarged representation for $0 \leq p_{z} \leq 1$ a.u. Figure reproduced from reference [48]. (This figure is subject to copyright protection and is not covered by a Creative Commons license.)

an excellent agreement with the experimental anisotropies. The limited momentum range may be due to the inherent DMFT approximation that the self energy neglects spatial fluctuations.

\subsubsection{Relativistic effects, moments of the Compton profiles and total energies}

There are a few theoretical methods of electronic structure available to quantitatively assess the interplay between correlation and relativistic effects. Within the framework of DFT the recently developed KKR+DMFT scheme demonstrates a clear advantage in this direction. From a pragmatic point of view perturbative solvers of DMFT written in adapted basis sets to include spin-orbit effects $[1,32]$ are efficient tools for realistic multi atom/orbital calculations. This means that we in fact capture the interplay between relativistic and correlation effects at a more economical level of the theory: from the correlations point of view a perturbative solver is considered, while for the relativistic part the four-component was replaced by a two component formulation. For a single-particle in an effective spherical symmetric potential $V_{\text {eff }}$, the most common transformation of the Dirac Hamiltonian into the two component formulation is expressed as a unitary transformation [66], that leads to the following series in power of $\propto 1 / c^{2}$ :

$H^{B P}=\left(m c^{2}+V_{e f f}+\frac{p^{2}}{2 m}\right)-\frac{p^{4}}{8 m^{3} c^{2}}-\frac{1}{8 m^{2} c^{2}}\left(p^{2} V_{e f f}\right)+\frac{\hbar}{4 m^{2} c^{2}} \sigma\left(\nabla V_{e f f} \times \mathbf{p}\right)+\ldots$

The first terms in parenthesis in equation (14) represent the usual non-relativistic Hamiltonian including the rest mass energy, the second one is the so called massvelocity term, the third is called the Darwin term and the fourth operator describes the spin-orbit coupling (interaction). It can be analytically proved that the scalar mass-velocity and Darwin terms are unbounded from below. The resulting Breit-Pauli Hamiltonian $\left(H^{B P}\right)$ is also known as the first order relativistic Hamiltonian. 
The measured Compton profile, or the momentum distribution enables in principle to obtain averages $\left\langle p^{n}\right\rangle$ directly from experiment. On the computational side, the momentum space formulation allows to obtain the Compton profiles within the $\operatorname{LSDA}(+\mathrm{U} / \mathrm{DMFT})[43,47,49]$ for many systems.

Further on additional information can be gained by taking moments of the difference between the correlated and non-correlated Compton profiles along different K-directions: $p_{z}|| \mathbf{K}$

$$
\left\langle p^{n}\right\rangle_{\mathbf{K}}=\int_{0}^{\infty} p_{z}^{n}\left[J_{\mathbf{K}}^{+U / D M F T}\left(p_{z}\right)-J_{\mathbf{K}}^{L S D A}\left(p_{z}\right)\right] d p_{z} .
$$

The second $(n=2)$ and the forth $(n=4)$ moments of the difference in the total Compton profiles, along the bond directions can be connected with some specific terms from the expansion (14). In the following we discuss the connection between the second moment of the difference between correlated (LSDA + DMFT) and non-correlated (LSDA) Compton profiles and the kinetic energy of the electronic system. Our main focus is on the bond average of the second moment of the difference Compton profiles:

$$
\overline{\left\langle p^{2}\right\rangle}=\frac{1}{N_{b}} \sum_{\mathbf{K}} \int_{0}^{\infty} p_{z}^{2} \Delta J_{\mathbf{K}}\left(p_{z}\right) d p_{z} \propto E_{k i n}^{D M F T}-E_{k i n}^{L S D A}
$$

The overbar represents the average taken over the bonds extending along the $\mathbf{K}$-directions, $\Delta J_{\mathbf{K}}\left(p_{z}\right)$ is the difference of the total Compton profiles, $N_{b}$ is the number of bonds. On the right hand side of equation (16) are the kinetic energies computed in DMFT/LSDA. In general, calculating total energies in LSDA + DMFT is a difficult task and requires the evaluation of an energy functional with several terms $[1,75]$ including the Galitskii-Migdal contribution [67], i.e., $1 / 2 \operatorname{Tr}[\hat{\Sigma} \hat{G}]$, and the double counting as well. The LSDA + DMFT total energy functional can in principle be analysed to obtain an expression for the kinetic energy similarly to what is done for DFT $[68,69]$. When focusing on the differences between LSDA + DMFT and LSDA, one can write:

$$
\Delta E_{k i n}=\operatorname{Tr}\left[\hat{H}_{K S}^{D M F T} \hat{G}^{D M F T}\right]-\operatorname{Tr}\left[\hat{H}_{K S}^{L S D A} \hat{G}^{L S D A}\right]+\left\langle\Delta V_{K S}\right\rangle+\left\langle\Delta T_{c}\right\rangle .
$$

In this expression the first and second terms on the right hand side are the single particle energies of the Kohn-Sham Hamiltonian within LSDA + DMFT and LSDA, while the third term is the expectation value of the difference of their corresponding Kohn-Sham potentials. The last term in equation (17) is the variation of the exchangecorrelation contribution to the kinetic energy, and can in principle be expressed in terms of the exchange-correlation potential and its gradient [69]. In Figure 4 we show the variation of the kinetic energy as obtained from equation (17) by ignoring the last term $\left\langle\Delta T_{c}\right\rangle$. These data were obtained through a full-potential linearized muffintin orbital (FP-LMTO) code $[70,76]$, which has been shown to give results in very good agreement with SPR-KKR regarding LSDA + DMFT total energies [75]. The second moments along the bonds $\left\langle p^{2}\right\rangle_{\mathbf{K}}$ are also shown in Figure 4, demonstrating that the electronic correlation energies are anisotropic. One has to note that the main source for the anisotropy in the momentum space is bond directionality that is already captured within the LSDA. However, this can not provide any measure for the electronic correlations.

In fact, from Figure 4 it is clear that the two contributions are still far from quantitative agreement. However, we capture a consistent qualitative picture pointing to a decrease of the kinetic energy difference for increasing $U$. In the low momentum 

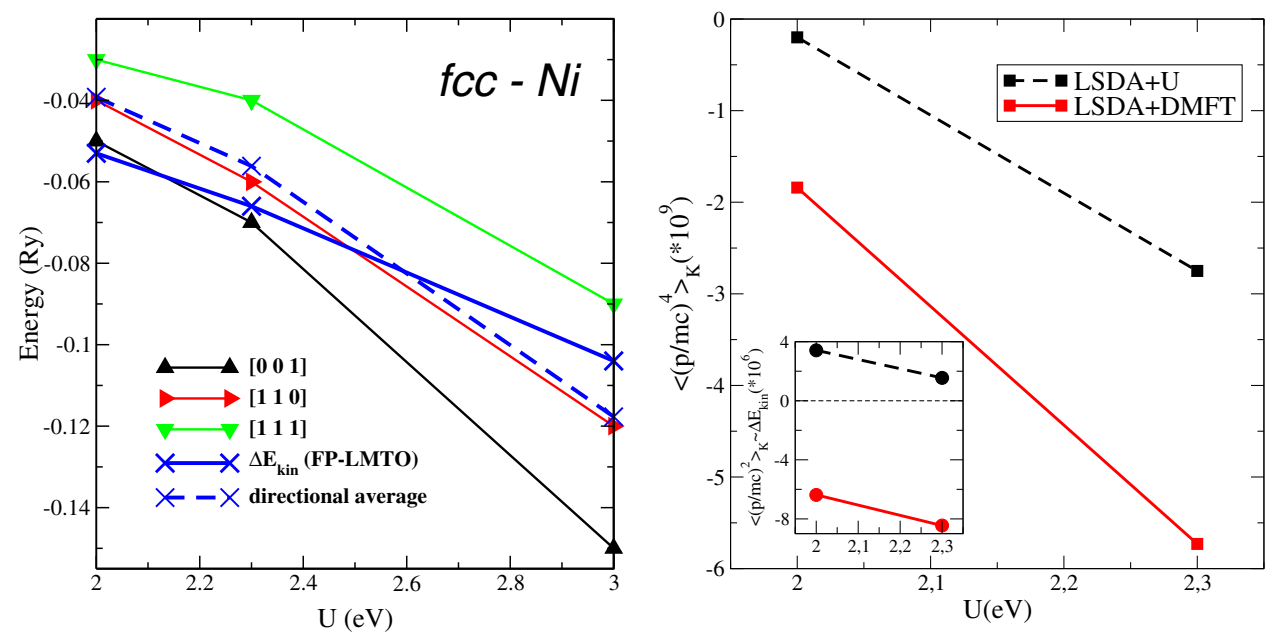

Fig. 4. Left panel: second moment of the difference profiles $\delta J_{\mathbf{K}}(p)$ along the symmetry directions [001] solid black, [110] read triangle, [111] green triangle. Directional average (blue dashed line), compared with the kinetic energy difference (solid blue line). Right panel: averaged forth and second moments of the Compton profiles computed within LSDA + DMFT (red solid) and LSDA+U (black solid). $U=2.0 / 2.3 \mathrm{eV}, J=0.9 \mathrm{eV}$ and $T=400 \mathrm{~K}$. Left panel reproduced form [47], right pannel [48]. (This figure is subject to copyright protection and is not covered by a Creative Commons license.)

region increasing the values of $U$ an increasing in the kinetic energy is obtained, which is in agreement with the argument that the presence of $U$ penalises the electrons and leads to an increase in their kinetic energy. This argument is not valid anymore in the region of high momenta, where the mean-field type exchange-correlation dominates the "Hubbard-U" contribution. Further analysis is needed in order to make a more quantitative comparison, especially to understand the role of the double counting correction and the effects of the expectation value of $T_{c}$, discarded in the present analysis.

We evaluate the so called free particle relativistic kinetic energy $\left(H_{0}\right)$ in terms of the second moments and its relativistic correction as the forth moment, along the bond directions of Ni. In order to compare the magnitude of second and fourth order moments one has to introduce a dimensionless quantity $p_{r}=p / m c$. In terms of this reduced variable $H_{0}=m c^{2}\left((1 / 2) p_{r}^{2}-(1 / 8) p_{r}^{4}+\ldots\right)$ and the relevant expectation values has the expression:

$$
\left\langle H_{0}\right\rangle_{\mathbf{K}}=\int H_{0} d p_{z} \Delta J_{\mathbf{K}}^{+U / D M F T}\left(p_{z}\right) \approx m c^{2}\left[\frac{1}{2}\left\langle p_{r}^{2}\right\rangle_{\mathbf{K}}-\frac{1}{8}\left\langle p_{r}^{4}\right\rangle_{\mathbf{K}}\right] ; \quad\left(p_{z} \| \mathbf{K}\right) .
$$

Although the fourth order moments are significantly smaller than that of second order, $\left\langle p_{r}^{4}\right\rangle \propto 10^{-3}\left\langle p_{r}^{2}\right\rangle$, an overall non-negligible contribution is obtained: The relativistic corrections to the kinetic energy (see Fig. 4) are both negative and we see that dynamical correlations (LSDA + DMFT) generate larger relativistic corrections to the one-particle kinetic energy in comparison to their mean field (LSDA + U) counter part. In the range of the studied values of $U$ qualitative and quantitative differences are seen in the Compton profiles depending weather the LSDA is supplemented with static or dynamical many-body corrections. An important message is that relativistic effects and electronic correlations may have a non-trivial interplay and dynamical correlations determine larger relativistic corrections in the electronic 

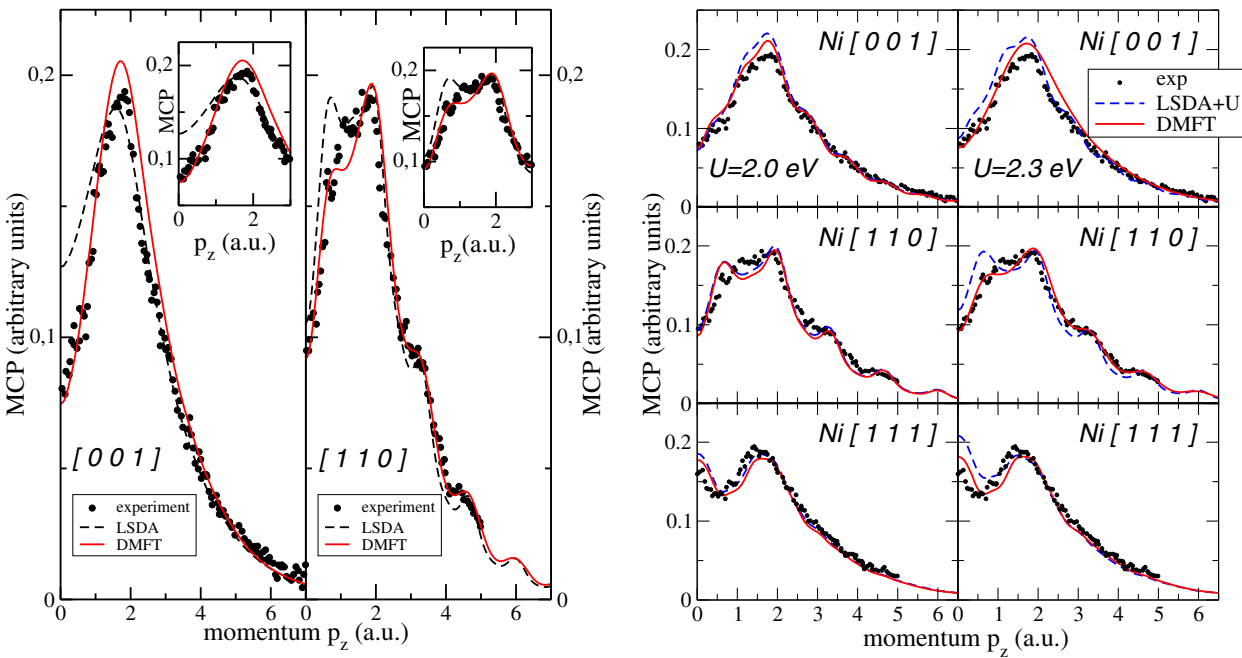

Fig. 5. Left panel: magnetic Compton profiles of Ni along [001] and [110] directions computed with LSDA and LSDA + DMFT with $U=2.3 \mathrm{eV}, J=0.9 \mathrm{eV}$. The computed data were convoluted according to the experimental resolution of $0.43 \mathrm{a}$.u. The experimental profiles are taken from reference [71]. Right panel: Compton profiles of $\mathrm{Ni}$ along the principal directions with experimental data reference [72].

structure of solids. Further investigations are necessary for a quantitative assessments of such effects.

\subsubsection{Magnetic Compton profiles of nickel}

The magnetic Compton profiles of $\mathrm{Ni}$ along the [001] and [110] directions are shown in Figure 5. The dashed (solid, red) curve represents results of the LSDA (LSDA + DMFT) calculations. The theoretical calculations are compared with the experimental MCP data [71,72]. The experimental momentum resolution is 0.43 a.u., which was also used as Gaussian broadening parameter for the calculated MCP spectra. In addition, the calculated MCPs have been scaled to the spin magnetic moment $\left(0.6 \mu_{B}\right.$ for both LSDA and LSDA + DMFT calculations).

As one can see in the left panel of Figure 5 the significant broadening takes away almost all fine structure features of the spectra. The LSDA results already show a reasonable agreement with the measurements. It captures the behaviour at large moments, it gets close to the maximum at $\approx 1.8$ a.u. and overestimates the low momentum region. In contrast to this, the DMFT reproduces significantly better the low momentum region, and the position of the maximum. However, it slightly overestimates the absolute value at the maximum and consequently deviates slightly from the experimental data in the intermediate region. The insets show the low momentum region. The right panel of Figure 5, shows the MCPs along the principal crystalographic directions. For all momenta the presence of correlations improves the agreement with experiment. In particular in the low momentum region $\left(p_{z}<1.5\right.$ a.u.).

According to our results, the mean field decoupling of the interaction $(+\mathrm{U})$ overestimates slightly the MCP spectra, while dynamic correlations improve the agreement with experiment. To reveal differences between the LSDA + U and LSDA + DMFT approaches we studied the directional differences, i.e., differences of Compton profiles with respect to the LSDA spectra. Overall the difference spectra follow a similar 
momentum dependence with visible deviations in the low momentum region. A qualitative difference is evidenced in this region: within the mean field approach $(+\mathrm{U})$ negative differences are seen while in the dynamic case, the opposite result is obtained. According to our suggested picture of momentum redistribution because of interaction [47] we conclude that the weight from low momentum distribution is shifted towards the higher momentum region in the LSDA + U spectra. This is in agreement with the naive picture of the effects of LSDA $+\mathrm{U}$ on the spectral weight distribution shifting weights towards higher energies. In the Compton scattering language, photons would be scattered accordingly by moving electrons situated in higher energy bands, although this does not mean that the electrons are moving faster, explaining the fact that there are no dramatic changes in the Compton spectra (differences of the order of \pm 0.02 ). On the contrary to the LSDA + U results, in the DMFT calculations the Fermi liquid type of self energy determines the spectral weight transfer towards the low energy region, and accordingly the spectra of photon scattering on the renormalized electronic structure would be redistributed towards low momenta. Similar conclusions have been reached in our previous studies $[43,47]$.

\subsection{Photoemission spectroscopy}

The most direct probe of the electronic structure of the valence band is provided by ARPES or its inverse version (IPE). An appropriate theoretical description of ARPES and IPE is supplied by the so called one-step model of photoemission. The main idea of it is to describe the photoemission process as a single coherent quantum-mechanical process. This approach includes more or less all relevant spectroscopy issues, like multiple-scattering events, matrix element, final state effects and surface effects. The approach goes back to the developments worked out by Pendry and coworkers [7779]. For calculation of the matrix elements that define the transition probability of the photoelectron, one has to consider the initial- and final-state wave functions. The final state has been constructed using the theory of spin-polarised low-energy electron diffraction (SPLEED). In this framework, the final state is represented by a so called time-reversed SPLEED state [77]. The initial valence band states are represented by the Green function. Both states are in practice calculated by means of the layer KKR theory [6]. Within this approach it is straight forward to describe complex layered semi-infinite structures, like surfaces, thin films or multilayers. Recent technical developments allow one to perform calculations of the ordered as well as chemically disordered systems including fully relativistic and electronic correlation effects $[3,22,46]$.

In the following, ferromagnetic transition metal systems are considered as prototype materials to study electronic correlations and magnetism beyond the LSDA. Various one-step model of ARPES studies including correlation effects by means of LSDA + DMFT have been performed. In particular, the spin dependent quasiparticle life-times and strength of electronic correlation effects in the ferromagnetic $3 d$ transition metals $(\mathrm{Fe}, \mathrm{Co}$ and $\mathrm{Ni}$ ) have been studied in a detail by spin- and angle-resolved photoemission $[20,39,40,80]$. A detailed comparison between theory and experiment is shown in Figure 6. Figure 7 shows the experimentally extracted imaginary part of the self energy in direct comparison with results based on the DMFT (using a pertrubative FLEX-solver) and an alternative many-body technique, the so called three body scattering (3BS) scheme [81]. A reasonable agreement with the spin-integrated as well as spin-resolved experimental data for all three surfaces and various photon-energies has been achieved. This agreement was previously not obtainable by LSDA calculations. Our quantitative analysis reveals that inclusion of local many-body Coulomb interactions within the LSDA + DMFT method are of ultimate importance for a realistic description of correlation effects in ferromagnetic 


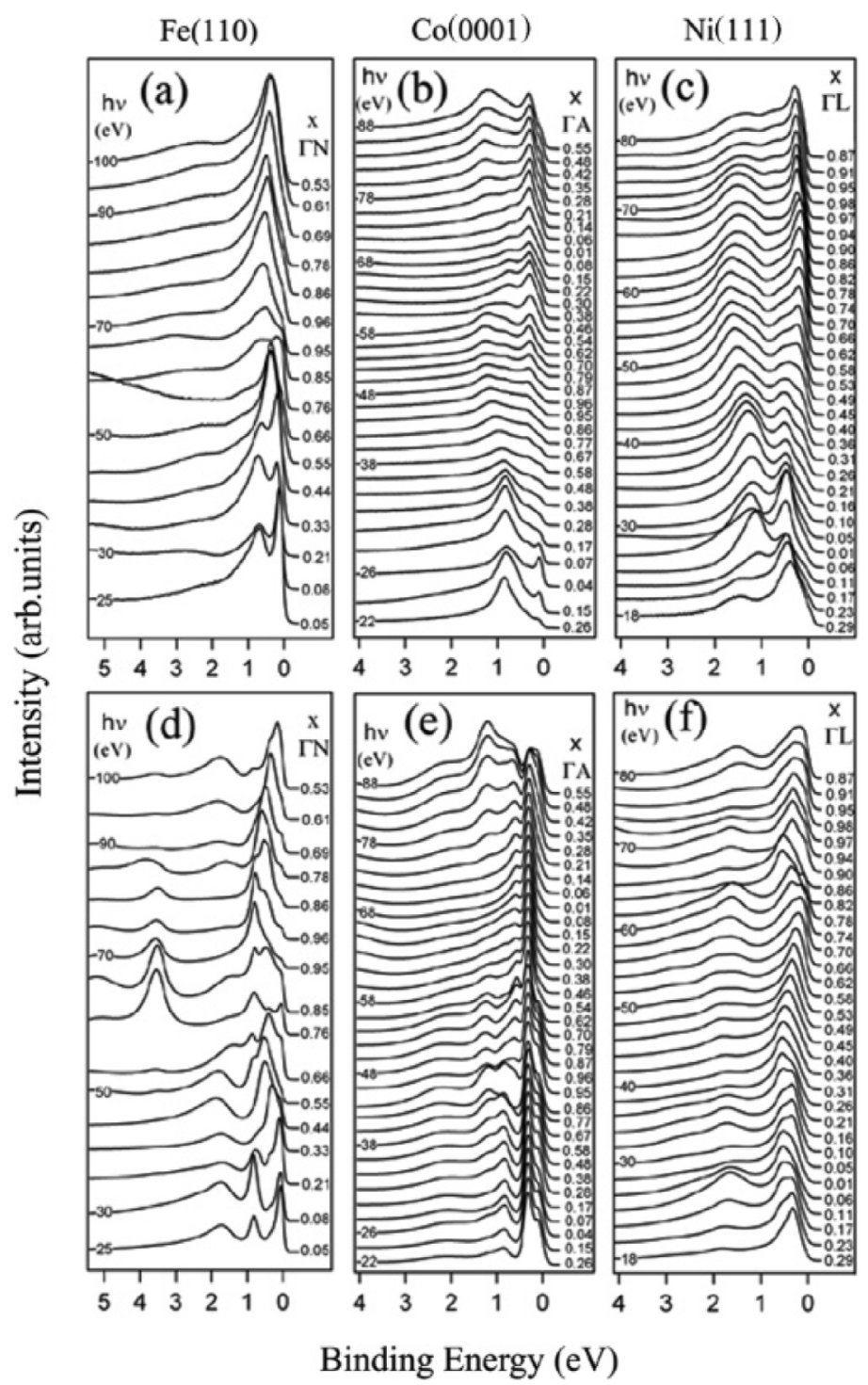

Fig. 6. Top: series of experimental spin-integrated photoemission spectra of (a) bcc Fe(110), (b) hcp Co(0001), and (c) fcc Ni(111). Left panel bottom: corresponding LSDA + DMFTbased calculations within the one-step model of photoemission for an in-plane magnetisation according to the experimental situation. Figure is reproduced from [80]. (This figure is subject to copyright protection and is not covered by a Creative Commons license.)

$3 d$ transition metals. In addition in Figure 6, several spectral features close to the Fermi level can be attributed to surface states and surface resonances. Although these states have predominantly surface character, their position in energy and dispersion is strongly influenced by DMFT which has been applied only to $d$-states. Similar effects can be seen in the inverse photoemission of fcc-Co(001) [41]. This study showed that surface effects play an important role and are necessary to be included into the LSDA + DMFT scheme. In the case of Fe(001) it turned out that the discrepencies between the experimental and theoretical spectra can be trace back to the non-local contributions to the self energy [40]. The increasing values of the on-site Coulomb 


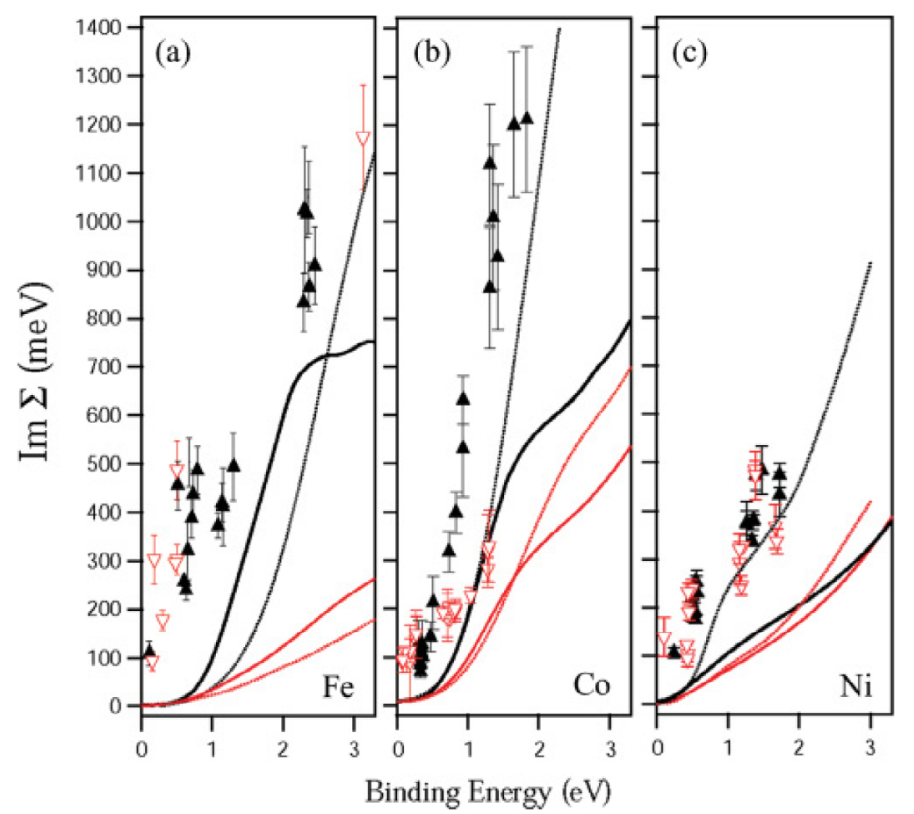

Fig. 7. Comparison between the experimental (symbols) and theoretical $\Im \Sigma$ (lines) for majority [black] and minority [red] spin electrons. The theoretical calculations correspond to $\Im \Sigma$ obtained from the DMFT (thick solid lines) and 3BS (thin dotted lines) calculations. Figure is reproduced from [80]. (This figure is subject to copyright protection and is not covered by a Creative Commons license.)

interaction parameter $U$ and the band narrowing of majority spin states, which were obtained when moving from $\mathrm{Fe}$ to $\mathrm{Ni}$, indicate that the effect of non-local correlations becomes weaker with increasing atomic number, whereas local correlation effects tend to be stronger.

Recently, the one-step model of photoemission has been extended to the soft-X-ray (sub-keV) and hard-X-ray (multi-keV) regime [82-84]. Increasing the photon energy means increasing the bulk sensitivity of the corresponding photoemission data and gives access to deeper lying layers in a sample. This technique has now been applied to a wide variety of materials including strongly correlated materials. Understanding the electronic and magnetic properties of diluted magnetic semiconductors, like e.g., Mn-doped GaAs, by the UV-ARPES has been a challenge due to the problems with the surface preparation (see [85] and references therein). The detailed understanding of the states near the Fermi level is necessary for explanation of the nature of ferromagnetic coupling. Two extreme limits have been discussed for explaining ferromagnetism in $(\mathrm{Ga}, \mathrm{Mn}) \mathrm{As}$. In one case $\mathrm{Mn} d$-states strongly hybridise with GaAs valence band states close to the Fermi level leading to ferromagnetism through $p$ - $d$ exchange. In the second model impurity bands are separated by $30-100 \mathrm{meV}$ from the valence band leading to double-exchange mechanism. Recently, this problem could be settled by angle resolved [18] and angle integrated [19] bulk sensitive hard X-ray photoemission. From the theoretical point of view, in order to describe this class of materials the computational scheme presented above has been extended to the combination of the exact diagonalisation solver [86] and CPA and therefore disorder is included naturally and on equal footing with the DMFT.

In Figure 8 we compare results of the one-step model of photoemission for GaAs and $\mathrm{Ga}_{0.97} \mathrm{Mn}_{0.03}$ As calculated within LSDA and a corresponding experimental hard-X-ray ARPES measured at $3.24 \mathrm{keV}$ [18]. Although overall agreement between 

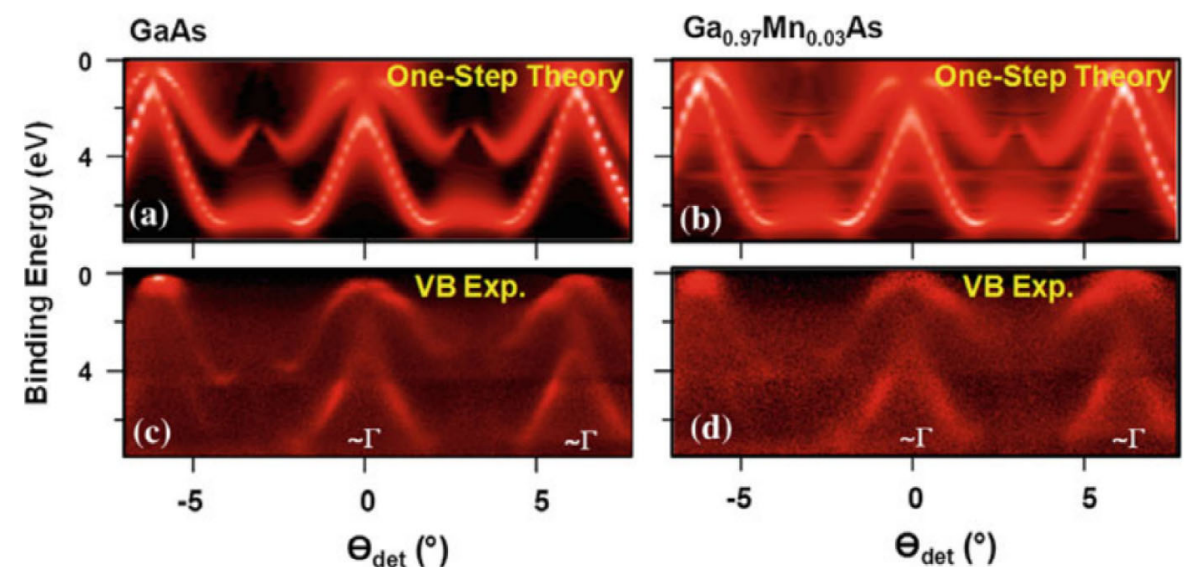

Fig. 8. (a) and (b) LSDA results of one-step hard-X-ray angle-resolved photoemission calculations for GaAs and $\mathrm{Ga}_{0.97} \mathrm{Mn}_{0.03}$ As respectively. (c) and (d) Experimental data measured at $3.24 \mathrm{keV}$. Figure reproduced from [18]. (This figure is subject to copyright protection and is not covered by a Creative Commons license.)
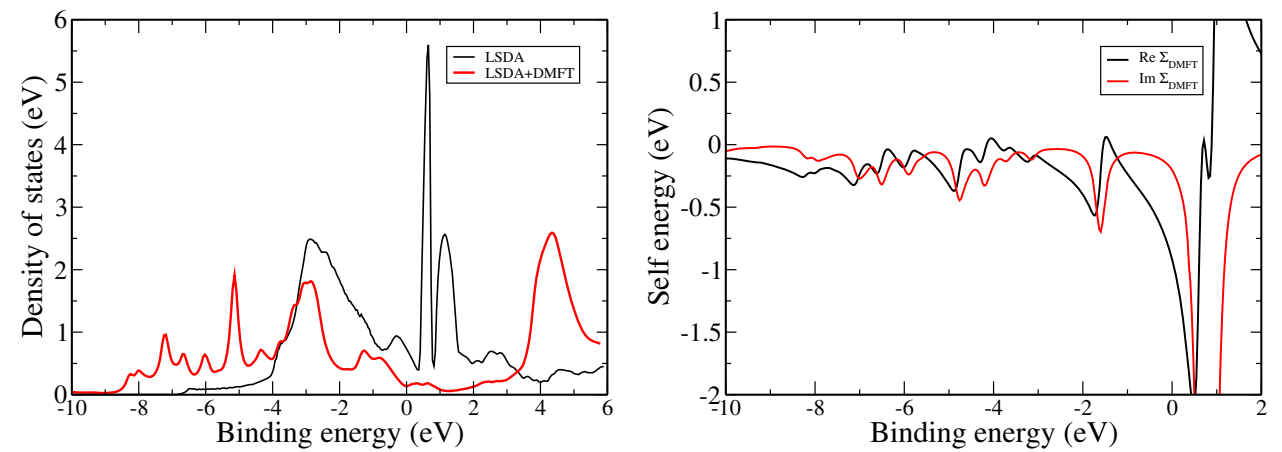

Fig. 9. Left: Mn $d$-like partial density of states calculated by the LSDA and LSDA + DMFT approach within the CPA. Right panel: real and imaginary part of the self energy calculated by exact diagonalisation calculated in the complex plane $0.1 \mathrm{eV}$ above real energy axis. The finite broadening of the imaginary part of self energy is artificial and is not related to the quasi-particle life time in the case of exact diagonalisation.

experiment and theory is remarkable, a closer look around the Fermi level reveals several inconsistencies. In particular, localised states of $\mathrm{Mn}$ are found at about $0.3 \mathrm{eV}$ below the Fermi level. This feature was not reproduced by the LSDA calculations. In order to understand these features, we performed additional LSDA + DMFT calculations [19] and corresponding concentration dependent angle integrated study. The exact diagonalisation self energy as shown in the right panel of Figure 9 has been calculated using the parameters described by DiMarco et al. [87]. The overall agreement between the resonant photoemission data [87] and the LDA+DMFT density of states shown in the left panel of Figure 9 is very good. The shift of the majority $d$-states of $\mathrm{Mn}$ to the lower binding energies at -3 and $-5 \mathrm{eV}$ is in agreement with previous LSDA $+\mathrm{U}$ calculations [88]. At low binding energies between -6 and $-10 \mathrm{eV}$ clear satellite structures appear. These structures can also be seen in the experiments. In Figure 10 we show experimental (a)-(c) and theoretical curves (d), (f) based on the one-step model of angle integrated photoemission. Our LDA + DMFT calculations agree quantitatively with the experimental data over a wide range of binding 

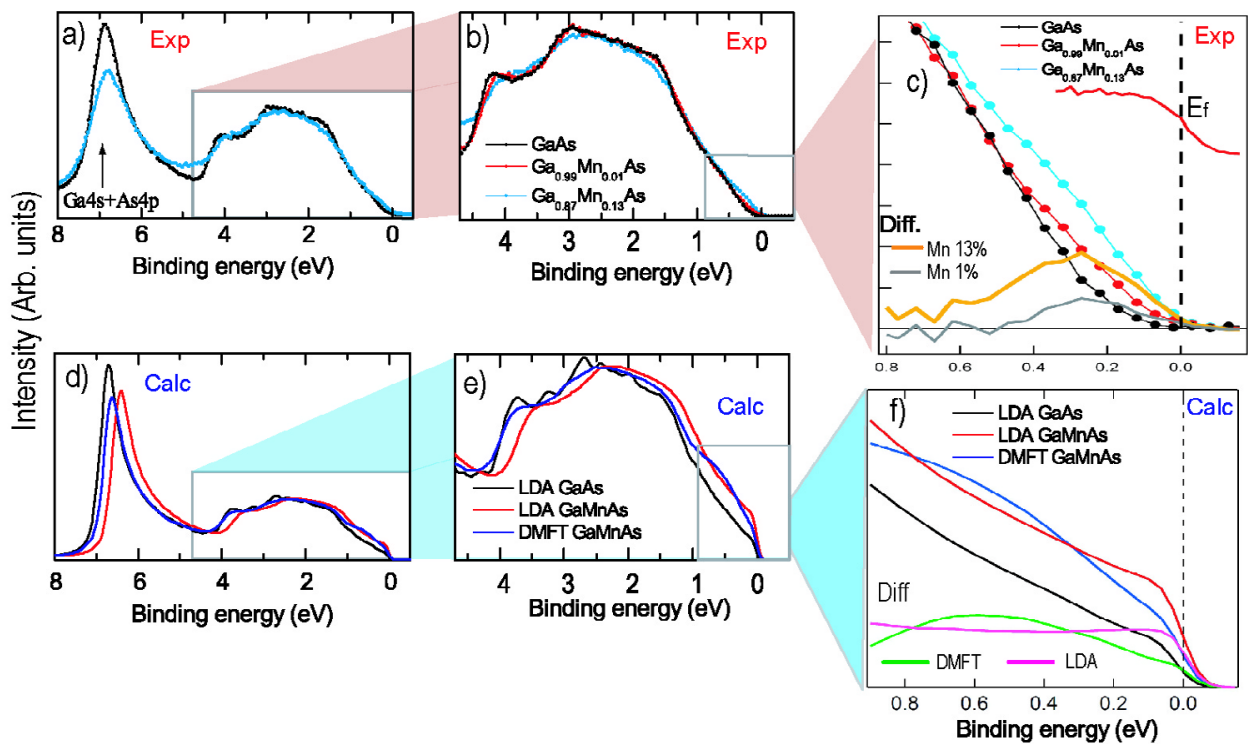

Fig. 10. (a), (b) and (c): experimental angle integrated hard-X-ray spectra for various Mn concentration in GaAs. (d), (e) and (f): corresponding theoretical one-step model data for $13 \%$ Mn using LDA (red curve) and LDA + DMFT (blue curve). Panel (c) and (f) show in addition the Mn-contribution to the spectral weight calculated as a difference between undoped and doped spectra. Figure reproduced from [19]. (This figure is subject to copyright protection and is not covered by a Creative Commons license.)

energies. In particular, we found close to Fermi level a peak with its maximum lying at $250 \mathrm{meV}$ below the Fermi edge, which is better described by LDA+DMFT than by LDA calculations. From the calculations we infer that the maximum in the difference signal has mainly $\mathrm{Mn}-3 d\left(\mathrm{t}_{2 g}\right)$ character. A strong hybridisation is present with a mixture of mainly As $4 p$ states localised around the impurity, and Mn $4 p$ states. It is important to emphasise that the Mn-related DOS near the Fermi edge, although significantly smaller, is nonzero within the energy resolution. This indicates that ferromagnetism in $(\mathrm{Ga}, \mathrm{Mn})$ As must be considered to arise from both $p$ - $d$ exchange and double-exchange, thus providing a unified picture of this controversial material.

\section{Summary}

In summary, we have reviewed an LSDA + DMFT implementation on the basis of the KKR band structure method. This combined scheme is particularly suitable to study the interaction of photons and electrons with matter. Nowadays, many technological applications such as materials characterization as well as electro-optical devices are based on the understanding of these interactions. In this area many fundamental questions concerning the theoretical / numerical aspects are still open.

We have presented the principles of the LSDA + DMFT method using the fully self-consistent, full potential, fully relativistic KKR formulation. This approach offers a clear physical picture and a straightforward analysis of spectroscopy results that are connected with the presence of the spin-orbit coupling. In our examples we rather followed at this particular point of view and looked at spin-orbit coupling induced properties of the ground state as well as the excited states. 
A systematic improvement of the actual implementation requires to work on both the KKR(LSDA) and DMFT sides. In order to compute and predict magnetocrystalline anisotropy an improved accuracy is required. The still open problem, include the need for a technical development to overcome the bottleneck linked to the analytical continuation of the Green function from the complex contour of KKR(LSDA) into the Matsubara axis where the impurity (DMFT) problem is solved. More success and failures of the analytical continuation (stochastic or in its actual form) are needed in order to sort out this question. Advancing this problem will also open the possibility to calculate within the Kubo formalism the anomalous Hall effect and related transport properties. Using a perturbative solver, as available in the current implementation, the calculations are less cumbersome. Better solvers, such as the exact diagonalization (ED) or the numerically exact continuous-time quantum Monte Carlo (CT-QMC) have still to be adapted to the KKR(LSDA), especially when this method is meant to be used for the calculation of multiplet effects in the absorption spectra (one-step model) of solids. As shown in our example (ARPES of Fe) it is expected that including non-local correlations into one-step model of photoemission by means of the combined GW and DMFT would further improve on the agreement with experimental results. In addition this will allow not only to get a proper description of the bulk states, but also the proper asymptotic behavior of the surface barrier.

Other problems that will be treated as a motivation for future research is briefly summarized as follows: (i) the description of disorder in correlated materials using Compton and Magnetic Compton scattering; (ii) The one step-model of photoemission in combination with DMFT, at equilibrium and (iii) its generalization to the pump-probe case, eventually the combination with the non-equilibrium DMFT. In conclusion is seems reasonable to suppose that progress will come form a common effort of people involved in the fields of Density Functional and Dynamical Mean Field Theory.

Support of the Deutsche Forschungsgemeinschaft through FOR 1346, the BMBF (05K16WMA) and CEDAMNF project (CZ.02.1.01/0.0/0.0/15_003/0000358) are acknowledged.

Open Access This is an Open Access article distributed under the terms of the Creative Commons Attribution License (http://creativecommons.org/licenses/by/4.0), which permits unrestricted use, distribution, and reproduction in any medium, provided the original work is properly cited.

\section{References}

1. G. Kotliar, S.Y. Savrasov, K. Haule, V.S. Oudovenko, O. Parcollet, C.A. Marianetti, Rev. Mod. Phys. 78, 865 (2006)

2. K. Held, Adv. Phys. 56, 829 (2007)

3. J. Minár, J. Phys.: Condens. Matter 23, 253201 (2011)

4. S.Y. Savrasov, G. Kotliar, Phys. Rev. B 69, 245101 (2004)

5. J. Minár, L. Chioncel, A. Perlov, H. Ebert, M.I. Katsnelson, A.I. Lichtenstein, Phys. Rev. B 72, 045125 (2005)

6. H. Ebert, D. Ködderitzsch, J. Minár, Rep. Prog. Phys. 74, 096501 (2011)

7. V. Drchal, V. Janiš, J. Kudrnovský, Phys. Rev. B 60, 15664 (1999)

8. S. Chadov, J. Minár, M.I. Katsnelson, H. Ebert, D.Ködderitzsch, A.I. Lichtenstein, Europhys. Lett. 82, 37001 (2008) 
9. O. Šipr, J. Minár, S. Mankovsky, H. Ebert, Phys. Rev. B 78, 144403 (2008)

10. O. Šipr, J. Minár, A. Scherz, H. Wende, H. Ebert, Phys. Rev. B 84, 115102 (2011)

11. M. Pickel, A.B. Schmidt, F. Giesen, J. Braun, J. Minár, H. Ebert, M. Donath, M. Weinelt, Phys. Rev. Lett. 101, 066402 (2008)

12. J. Minr, S. Mankovsky, O. ipr, D. Benea, H. Ebert, J. Phys.: Condens. Matter 26, 274206 (2014)

13. I. Di Marco, J. Minár, J. Braun, M.I. Katsnelson, A. Grechnev, H. Ebert, A.I. Lichtenstein, O. Eriksson, Eur. Phys. J. B 72, 473 (2009)

14. I. Di Marco, J. Minár, S. Chadov, M.I. Katsnelson, H. Ebert, A.I. Lichtenstein, Phys. Rev. B 79, 115111 (2009)

15. J. Braun, J. Minár, F. Matthes, C.M. Schneider, H. Ebert, Phys. Rev. B 82, 024411 (2010)

16. S. Chadov, G.H. Fecher, C. Felser, J. Minár, J. Braun, H. Ebert, J. Phys. D: Appl. Phys. 42, $084002(2009)$

17. J.P. Wüstenberg, R. Fetzer, M. Aeschlimann, M. Cinchetti, J. Minár, J. Braun, H. Ebert, T. Ishikawa, T. Uemura, M. Yamamoto, Phys. Rev. B 85, 064407 (2012)

18. A.X. Gray, J. Minár, S. Ueda, P.R. Stone, Y. Yamashita, J. Fujii, J. Braun, L. Plucinski, C.M. Schneider, G. Panaccione, et al., Nat. Mater. 11, 957 (2012)

19. J. Fujii, B.R. Salles, M. Sperl, S. Ueda, M. Kobata, K. Kobayashi, Y. Yamashita, P. Torelli, M. Utz, C.S. Fadley, et al., Phys. Rev. Lett. 111, 097201 (2013)

20. J. Sánchez-Barriga, J. Minár, J. Braun, A. Varykhalov, V. Boni, I. Di Marco, O. Rader, V. Bellini, F. Manghi, H. Ebert, et al., Phys. Rev. B 82, 104414 (2010)

21. M. Kronseder, J. Minár, J. Braun, S. Günther, G. Woltersdorf, H. Ebert, C.H. Back, Phys. Rev. B 83, 132404 (2011)

22. J. Minár, J. Braun, S. Mankovsky, H. Ebert, J. Electron. Spectrosc. Relat. Phenom. 184, 91 (2011)

23. M.E. Rose, Relativistic Electron Theory (Wiley, New York, 1961)

24. P. Strange, J. Staunton, B.L. Gyorffy, J. Phys. C: Solid State Phys. 17, 3355 (1984)

25. H. Ebert, B.L. Gyorffy, J. Phys. F: Met. Phys. 18, 451 (1988)

26. B.L. Györffy, M.J. Stott, Band Structure Spectroscopy of Metals and Alloys (Academic Press, New York, 1973), p. 385

27. A. Gonis, W.H. Butler, Multiple scattering in solids, Graduate Texts in Contemporary Physics (Springer, Berlin, 1999)

28. P. Weinberger, Electron Scattering Theory for Ordered and Disordered Matter (Oxford University Press, Oxford, 1990)

29. H. Ebert, Fully Relativistic Band Structure Calculations for Magnetic Solids - Formalism and Application, in Electronic Structure and Physical Properties of Solids, edited by H. Dreyssé (Springer, Berlin, 2000), Vol. 535 of Lecture Notes in Physics, p. 191

30. E. Tamura, Phys. Rev. B 45, 3271 (1992)

31. S.H. Vosko, L. Wilk, M. Nusair, Can. J. Phys. 58, 1200 (1980),

32. L.V. Pourovskii, M.I. Katsnelson, A.I. Lichtenstein, Phys. Rev. B 72, 115106 (2005)

33. B. Glaubitz, S. Buschhorn, F. Brüssing, R. Abrudan, H. Zabel, J. Phys.: Condens. Matter 23, 254210 (2011)

34. P.H. Dederichs, R. Zeller, H. Akai, H. Ebert, J. Magn. Magn. Mater. 100, 241 (1991)

35. I.V. Solovyev, A.I. Liechtenstein, K. Terakura, Phys. Rev. Lett. 80, 5758 (1998)

36. I.V. Solovyev, Phys. Rev. Lett. 95, 267205 (2005)

37. S. Chadov, J. Minár, H. Ebert, A. Perlov, L. Chioncel, M.I. Katsnelson, A.I. Lichtenstein, Phys. Rev. B 74, R140411 (2006)

38. J. Minár, H. Ebert, C. De Nadaï, N.B. Brookes, F. Venturini, G. Ghiringhelli, L. Chioncel, M.I. Katsnelson, A.I. Lichtenstein, Phys. Rev. Lett. 95, 166401 (2005)

39. J. Braun, J. Minár, H. Ebert, M.I. Katsnelson, A.I. Lichtenstein, Phys. Rev. Lett. 97, $227601(2006)$

40. J. Sánchez-Barriga, J. Fink, V. Boni, I. Di Marco, J. Braun, J. Minár, A. Varykhalov, O. Rader, V. Bellini, F. Manghi, et al., Phys. Rev. Lett. 103, 267203 (2009)

41. T. Allmers, M. Donath, J. Braun, J. Minár, H. Ebert, Phys. Rev. B 84, 245426 (2011) 
42. J. Braun, J. Minár, H. Ebert, A. Chainani, J. Miyawaki, Y. Takata, M. Taguchi, M. Oura, S. Shin, Phys. Rev. B 85, 165105 (2012)

43. D. Benea, J. Minár, L. Chioncel, S. Mankovsky, H. Ebert, Phys. Rev. B 85, 085109 (2012)

44. K. Held, I.A. Nekrasov, G. Keller, V. Eyert, N. Blümer, A.K. McMahan, R.T. Scalettar, T. Pruschke, V.I. Anisimov, D. Vollhardt, Phys. Stat. Sol. (b) 243, 2599 (2006)

45. F. Manghi, V. Bellini, J. Osterwalder, T.J. Kreutz, P. Aebi, C. Arcangeli, Phys. Rev. B 59, R10409 (1999)

46. J. Braun, Rep. Prog. Phys. 59, 1267 (1996)

47. L. Chioncel, D. Benea, H. Ebert, I. Di Marco, J. Minár, Phys. Rev. B 89, 094425 (2014)

48. L. Chioncel, D. Benea, S. Mankovsky, H. Ebert, J. Minar, Phys. Rev. B 90, 184426 (2014)

49. M J Cooper, Rep. Prog. Phys. 48, 415 (1985)

50. D. Benea, Theoretical Description of Magnetic Compton Scattering and Magnetic Properties of Cr-chalcogenide Compounds, PhD thesis, LMU München, 2004

51. S. Wakoh, Y. Kubo J, Magn. Magn. Mater. 5, 202 (1977)

52. T. Baruah, R.R, Zope, A. Kshirsagar, Phys. Rev. B 62, 16435 (2000)

53. M. Tokii, M. Matsumoto, J. Phys.: Condens. Matter 18, 3639 (2006)

54. Y. Kubo, J. Phys. Chem. Solids 65, 2077 (2004)

55. J. Rath, C.S. Wang, R.A. Tawil, J. Callaway, Phys. Rev. B 8, 5139 (1973)

56. L. Lam, P.M. Platzman, Phys. Rev. B. 9, 5122 (1974)

57. L. Lam, P.M. Platzman, Phys. Rev. B. 9, 5128 (1974)

58. S. Wakoh, M. Matsumoto, J. Phys.: Condens. Matter 2, 797 (1990)

59. G.E.W. Bauer, J.R. Schneider, Z. Phys. B 54, 17 (1983)

60. G.E.W. Bauer, Phys. Rev. B 30, 1010 (1984)

61. Y. Kubo, J. Phys. Chem. Sol. 62, 2199 (2001)

62. P. Eisenberger, W.A. Reed, Phys. Rev. B 9, 3242 (1974)

63. C.S. Wang, J. Callaway, Phys. Rev. B 11, 2417 (1975)

64. D.L. Anastassopoulos, G.D. Priftis, N.I. Papanicolau, N.C. Bacalis, D.A. Papaconstantopoulos, J. Phys.: Condens. Matter 3, 1099 (1990)

65. A.J. Rollason, J.R. Schneider, D.S. Laundy, R.S. Holt, M.J. Cooper, J. Phys. F: Met. Phys. 17, 1105 (1987)

66. L.L. Foldy, S.A. Wouthuysen, Phys. Rev. 78, 29 (1950)

67. V.M. Galitskii, A.B. Migdal, Sov. Phys. J.E.T.P. 7, 96 (1958)

68. L. Mel, J.P. Perdew, Phys. Rev. A 32, 2010 (1985)

69. I.J. Rodríguez, J.W. Ayers, P.W. Götz, A.F.L. Castillo-Alvarado, J. Chem. Phys. 131, $0210101(2009)$

70. O. Granas, I. di Marco, P. Thunström, L. Nordström, O. Eriksson, T. Björkman, J.M. Wills, Comp. Mater. Science 55, 295 (2012)

71. Y. Sakurai, Y, Tanaka, T. Ohata, Y. Watanabe, S. Nanao, Y, Ushigami, T. Iwazumi, H. Kawata, N. Shiotani, J. Phys.: Condens. Matter 6, 9469 (1994)

72. M.A.G. Dixon, J.A. Duffy, S. Gardelis, J.E. McCarthy, M.J. Cooper, S.B. Dugdale, T. Jarlborg, D.N. Timms, J. Phys.: Condens. Matter 10, 2759 (1998)

73. Z. Szotek, B.L. Gyorffy, G.M. Stocks, W.M. Temmerman, J. Phys. F: Met. Phys. 14, $2571(1984)$

74. D. Benea, S. Mankovskyy, H. Ebert, Phys. Rev. B 73, 94411 (2006)

75. R.D. McNorton, J.M. MacLaren, J. Phys.: Condens. Matter 21, 445803 (2009)

76. A. Grechnev, I. Di Marco, M.I. Katsnelson, A.I. Lichtenstein, J. Wills, O. Eriksson, Phys. Rev. B 76, 035107 (2007)

77. J.B. Pendry, Low Energy Electron Diffraction (Academic Press, London, 1974)

78. J.F.L. Hopkinson, J.B. Pendry, D.J. Titterington, Comp. Phys. Commun. 19, 69 (1980)

79. J.B. Pendry, Surf. Sci. 57, 679 (1976)

80. J. Sánchez-Barriga, J. Braun, J. Minár, I. Di Marco, A. Varykhalov, O. Rader, V. Boni, V. Bellini, F. Manghi, H. Ebert, et al., Phys. Rev. B 85, 205109 (2012)

81. C. Calandra, F. Manghi, Phys. Rev. B 50, 2061 (1994) 
82. A.X. Gray, C. Papp, S. Ueda, B. Balke, Y. Yamashita, L. Plucinski, J. Minár, J. Braun, E.R. Ylvisaker, C.M. Schneider, et al., Nat. Mater. 10, 759 (2011)

83. C.S. Fadley, Synchrotron Radiation News 25, 26 (2012)

84. V.N. Strocov, T. Schmitt, U. Flechsig, T. Schmidt, A. Imhof, Q. Chen, J. Raabe, R. Betemps, D. Zimoch, J. Krempasky, et al., J. Synchr. Rad. 17, 631 (2010)

85. T. Dietl, H. Ohno, Rev. Mod. Phys. 86, 187 (2014)

86. P. Thunström, I. Di Marco, O. Eriksson, Phys. Rev. Lett. 109, 186401 (2012)

87. I. Di Marco, P. Thunström, M.I. Katsnelson, J. Sadowski, K. Karlsson, S. Lebègue, J. Kanski, O. Eriksson, Nat. Commun. 4, 951 (2013)

88. K. Sato, L. Bergqvist, J. Kudmovsky, P.H. Dederichs, O. Eriksson, I. Turek, B. Sanyal, G. Bouzerar, H. Katayama-Yoshida, V.A. Dinh, et al., Rev. Mod. Phys. 82, 1633 (2010) 